\title{
An incompletely penetrant novel $M A F B$ (p.Ser56Phe) variant in autosomal dominant multicentric carpotarsal osteolysis syndrome
}

\author{
GABRIEL C. DWORSCHAK ${ }^{1}$, MARKUS DRAAKEN ${ }^{1,2}$, ALINA HILGER $^{1}$, MARK BORN $^{3}$, \\ HEIKO REUTTER $^{1,4}$ and MICHAEL LUDWIG ${ }^{5}$ \\ ${ }^{1}$ Institute of Human Genetics, ${ }^{2}$ Department of Genomics, Life and Brain Center, ${ }^{3}$ Department of Pediatric Radiology, \\ Children's Hospital, ${ }^{4}$ Department of Neonatology and ${ }^{5}$ Department of Clinical Chemistry \\ and Clinical Pharmacology, University Hospital of Bonn, D-53127 Bonn, Germany
}

Received February 14,2013; Accepted April 16, 2013

DOI: 10.3892/ijmm.2013.1373

\begin{abstract}
Multicentric carpotarsal osteolysis syndrome (MCTO) is a rare autosomal dominant skeletal dysplasia usually presenting in early childhood with variable phenotypic features and course. Clinical manifestations comprise aggressive osteolysis of the carpal and tarsal bones in particular, an often progressive nephropathy leading to end-stage renal disease, craniofacial anomalies and mental impairment. Recently, heterozygous missense mutations in the V-maf musculoaponeurotic fibrosarcoma oncogene homolog $\mathrm{B}$ (avian) $(M A F B)$ gene have been causally related to MCTO patients in 13 unrelated families investigated. Contrary to these findings suggesting complete penetrance, in the present study, we identified a novel missense $M A F B$ variant present not only in the patient, but also in his unaffected mother, sister and maternal grandmother. This observation demonstrates an incomplete penetrance for some $M A F B$ mutations, thereby suggesting that modifier genes, epigenetic mechanisms or environmental factors may modulate the MCTO phenotype. This should be considered in diagnosis and genetic counseling.
\end{abstract}

\section{Introduction}

Since the first description of a 'disappearing bone disease' (1) several rare congenital osteolysis syndromes have been described with their nosology being classified by Tyler and Rosenbaum (2) in 1976 and by the International Classification of Constitutional Disorders of Bone (3). Autosomal dominant multicentric carpotarsal osteolysis syndrome (MCTO; OMIM 166300) usually presents in early childhood, often with a clinical appearance mimicking juvenile idiopathic

Correspondence to: Professor Michael Ludwig, Department of Clinical Chemistry and Clinical Pharmacology, University Hospital of Bonn, Sigmund-Freud-Str. 25, D-53127 Bonn, Germany

E-mail: mludwig@uni-bonn.de

Key words: carpal bones, osteolysis, skeletal dysplasia, tarsal bones, V-maf musculoaponeurotic fibrosarcoma oncogene homolog B (avian) gene, incomplete penetrance, modifier gene arthritis (JIA) (4). However, JIA can easily be differentiated from MCTO by clinical, laboratory and radiological findings (5). MCTO is characterized by progressive and most often bilateral destruction of the carpal and tarsal bones; however, osteolysis may also affect other bones. Affected individuals may develop proteinuria and progressive nephropathy leading to end-stage renal disease. Craniofacial anomalies and mental impairment have also been observed $(5,6)$. Recently, Zankl et al (7) reported an association of MCTO with de novo missense mutations in the V-maf musculoaponeurotic fibrosarcoma oncogene homolog $\mathrm{B}$ avian) $(M A F B)$ gene in 11 simplex cases. In two further multiplex families, $M A F B$ missense mutations segregated in an autosomal dominant fashion. The protein encoded (MafB) by this gene is a basic domain leucine zipper transcription factor with an anti-osteoclastogenic function also involved in renal tubule survival, tissue differentiation within the pancreas and segmentation of the hindbrain (8-13). The disturbance of these different functions may explain the phenotypic features observed in association with MCTO.

In the present study, we identified a novel p.Ser56Phe $M A F B$ missense variant in a German individual with MCTO. Surprisingly, an analysis of the family revealed presence of this variant in three unaffected female family members investigated.

\section{Patients and methods}

Patient description. The index case in this study is the second child of unrelated parents. He has a three years older healthy sister. At his first visit to the Genetics Department, University Hospital of Bonn, Bonn, Germany, the index case was 17 years of age. His early development was completely uneventful. By the age of four, he first visited the Children's Hospital for recurrent pain in his right hand with swelling of his wrist, raising the suspicion of the onset of juvenile rheumatoid arthritis. At the age of six he presented with dysesthesia in his right leg with swelling of his ankle. Subsequent diagnostic radiographic workup revealed several carpotarsal osteolytic spots. Extensive diagnostic testing did not reveal any immunological or infectious origin for his symptoms. During the course of his extensive diagnostic workup to explain his carpotarsal osteolysis, an electroencephalography revealed slow waves 
on his right temporo-parieto-occipital recordings. Subsequent brain magnetic resonance imaging revealed an Arnold-Chiari malformation type I. During late puberty he developed androgenetic alopecia and slight non-immunological bilateral exophthalmos. At 19 years of age, an X-ray revealed irregular and dysplastic, partially missing carpalia and dysplastic changes of the tarsalia (Fig. 1). Renal functional studies and urine analysis showed normal renal function, without any sign of proteinuria or nephropathy.

The mother did not report any history of swelling of her wrists or ankles nor any radiographical signs of carpotarsal osteolysis. In addition she did not present with any ulnar deviation of her hands or wrist arthralgia. X-ray images, renal functional analyses and urine analysis were normal. The sister and maternal grandmother also showed no features characteristic of MCTO.

Sample collection and DNA analysis. After informed consent was obtained, EDTA blood samples were obtained from the family members. Genomic DNA was isolated using the QIAamp DNA Blood kit and amplification of the single $M A F B$ exon was performed by polymerase chain reaction (PCR) using HotStarTaq DNA polymerase (Qiagen, Hilden, Germany). PCR primers (1F, 5'-GCTTGGCTCGGCGCGCTCC-3'; 1F2, 5'-CCGTGCGCGAGCTGAACCG-3'; 1R, 5'-GCGGCAGGG ACAGGGTCCG-3'; 1R2, 5'-TGCGAGCCGATGAGCGC TTC-3') also served as sequencing primers. For mutational analysis, PCR-amplified DNA products were subjected to direct automated sequencing using a 3130xl Genetic Analyzer (Applied Biosystems, Inc., Foster City, CA, USA) and both strands from the amplicons of the parents and patients were analyzed.

RNA analysis. RNA analyses were performed to investigate the expression of both alleles in the patient and his mother. Blood samples were collected in PAXgene tubes (PreAnalytiX, Hombrechtikon, Switzerland) and RNA for reverse transcription (RT)-PCR was prepared using the RNeasy Plus Micro kit (Qiagen) in accordance with the manufacturer's instructions. Allele-specific amplification (ASA)-PCR and allele-specific quantitative PCR (AS-qPCR) were carried out as previously described (14). ASA-PCR may lack reliability since the mutated primer may still allow amplification of the normal allele as shown by Hezard et al (15). These authors showed that allele discrimination can be improved by incorporating an additional mismatch in the antepenultimate position by substituting the respective wild-type (WT) pyrimidine (purine) nucleotide by the respective pyrimidine (purine) base. Therefore, to adjust for similar annealing temperatures, ASA forward (F) primers and a universal reverse (UR) and forward (UF) primer were designed as follows (normal WT sequence, 5'-TGGAACTG AAAACCAGCGACCA-3'): WT-F, 5'-GAACTGAAAAC CAGCGATCA-3'; mutant-G-F, 5'-GAACTGAAAACCAGC GATCG; UR, 5'GGCACTAACCTCTCAATGCTG-3'; and UF, 5'-TGGAACTGAAAACCAGCGACC-3'. Reactions for AS-qPCR were performed on an ABI PRISM 7900HT Fast Real-Time PCR System (Applied Biosystems, Inc.) using SYBR-Green for detection. Each assay was performed as previously described (14) and included DNA from four controls (two male and two female samples) and the subjects' DNA.
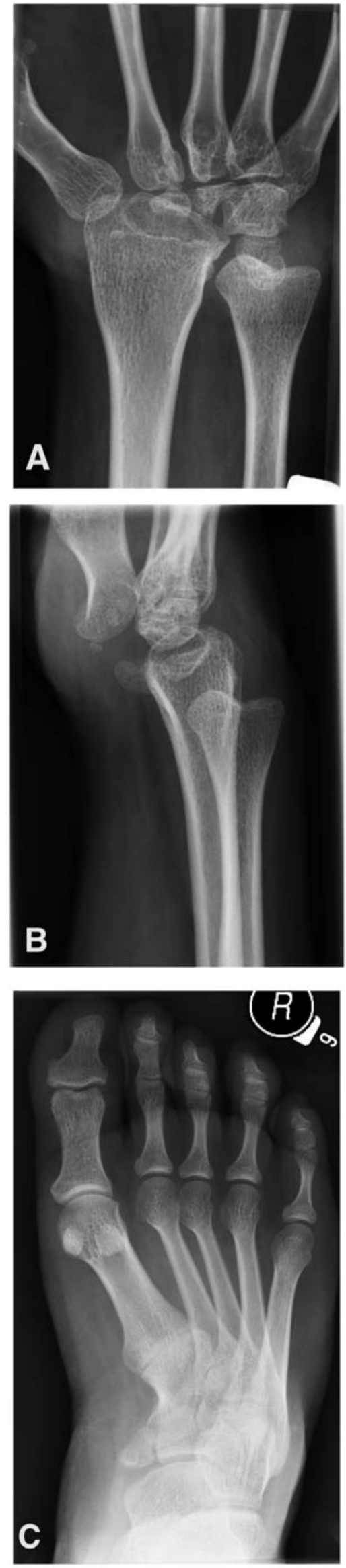

Figure 1. X-ray of the right hand and foot at the age of 19 years shows (A) irregular and dysplastic, partially missing carpalia and (B) a bayonet-like wrist deformity with palmar subluxation. (C) Dysplastic changes of the tarsalia with consequent malposition of the metatarsal bones are less pronounced. 
A

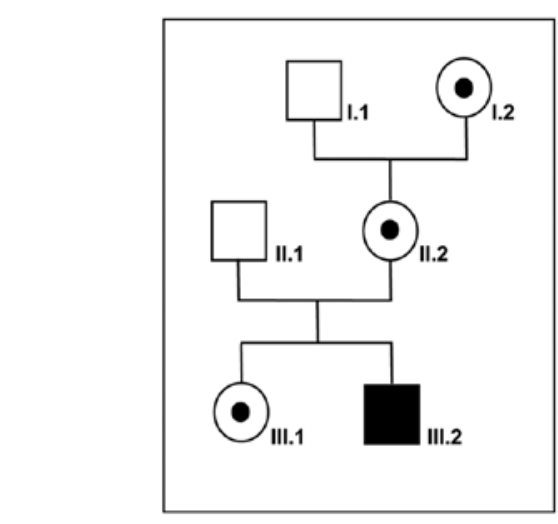

B

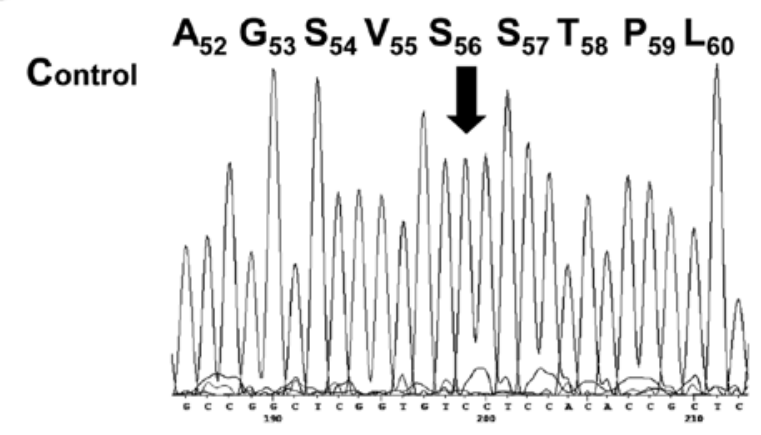

I.2, II.2, III.1,

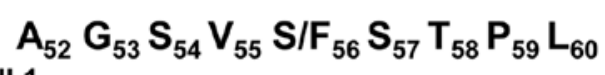

C

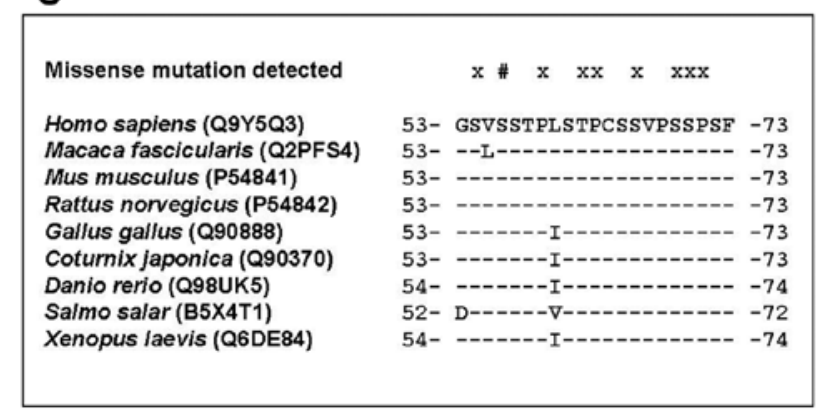

III.2

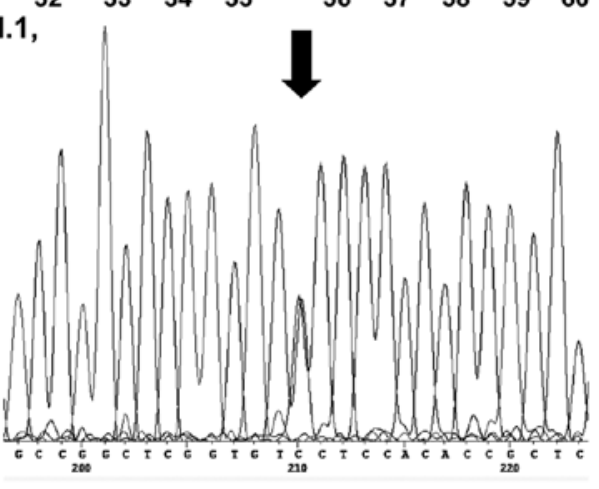

Figure 2. (A) Pedigree of the family investigated and (B) sequence analysis showing part of the V-maf musculoaponeurotic fibrosarcoma oncogene homolog B (avian) $(M A F B)$ gene compared with the control. The p.Ser56Phe variant detected in the multicentric carpotarsal osteolysis syndrome (MCTO) affected individual, his mother, sister and maternal grandmother is indicated by an arrow with the amino acids encoded given above. (C) Comparative genomics revealed the conservation of serine residue 56 in the transactivation domain of the MafB protein. Amino acid sequence alignment with the corresponding numbering is given with identical residues shown by dashes. Protein data were obtained from Swiss-Prot with the respective entry given in brackets. Amino acid substitutions detected by Zankl et al (7) (x) and the pSer56Phe substitution observed in our patient (\#) are indicated above.

\section{Results}

The screening of $M A F B$ PCR products obtained from the index case (Fig. 2A, III.2) revealed a single heterozygous c.167C $>\mathrm{T}$ nucleotide transition (Fig. 2B), predicting a p.Ser56Phe substitution (GenBank accession no. NM_005461.3, with the A of the start ATG as nucleotide 1). Gene analysis in the unaffected mother of the patient (II.2) provided evidence that she is a carrier of the mutant allele transmitted to her son. Moreover, the healthy sister and the healthy maternal grandmother also showed the heterozygous c.167C $>\mathrm{T}$ transition.

These findings led us to perform functional analyses. To exclude the sole or predominant expression of the WT allele in the mother, the RT-PCR products were first analyzed by sequence analysis. Fluorescence intensity indicated the presence of the WT and variant transcript in similar amounts in the samples of the patient and his mother. To verify this finding we also performed AS-qPCR.

Prior to $\mathrm{qPCR}$ it had to be validated that the ASA primers only direct amplification on their complementary allele and that the conditions chosen avoid any other unspecific amplification. The ASA primers designed allowed the sole amplification of the respective allele without any additional unspecific priming, hence, qPCR was performed. This method involves PCR amplification of the product of interest and, in a separate reaction, amplification of an invariantly expressed internal standard (housekeeping gene) to be used as a denominator to normalize the amount of the target gene. Allele-specific qPCR also showed similar amounts of both transcribed alleles in the patient and his mother (data not shown).

Several clues point to a critical role for serine 56 located in the amino-terminal transcriptional activation domain of MAFB: First, the c.167C $>\mathrm{T}$ transition has not been deposited in the single nucleotide polymorphism (SNP, Build137) database and in the Deep Catalog of Human Genetic Variation (www.1000genomes.org), which argues against the possibility that this substitution is just a rare benign variant. In addition, the amino acid exchange substitutes a polar residue for a nonpolar one and pathogenicity prediction with several algorithms (PolyPhen-2, SNPs\&GO, PMut, SIFT) predicted this variant to be disease-causing or at least possibly damaging. Finally, Ser56 is absolutely conserved at its corresponding position in species orthologues as part of the transactivation motif at least as far down as Xenopus laevis (X. laevis) (Fig. 2C).

\section{Discussion}

The patient presented in this study showed the typical features of MCTO with wrist and ankle swelling, severe osteolysis of almost all carpal and tarsal bones (Fig. 1) and bilateral exoph- 
thalmos. He showed no proteinuria or nephropathy at the time of writing, but it is as yet unclear, whether his renal function will remain normal. However, complete absence of renal involvement has been frequently observed (6) and was not reported in $50 \%$ of the patients reported by Zankl et al (7). We further noted Arnold-Chiari malformation type I and androgenetic alopecia but are not aware of any other case showing the coincidence of MCTO with these features.

The recent observations of Zankl et al (7) suggested a heterozygous de novo MAFB mutation in our isolated MCTO patient. As expected, we observed a single heterozygous p.Ser56Phe amino acid substitution that surprisingly was also present in three unaffected females in this family. $M A F B$ RNA analysis in the patient and his mother revealed similar amounts of mutant and WT mRNAs providing no evidence, that the phenotypic differences are a result of a preferential transcription of the WT allele in the mother.

Nevertheless, several strands of evidence suggested that the amino acid substitution observed represents a pathogenic mutation. Aside from its absence in databases, comparative genomics revealed apparent evolutionarily conservation of Ser56 at least as far down as X. laevis (Fig. 2C). In addition, pathogenicity prediction revealed this substitution to be disease-causing or at least possibly damaging. Moreover, this novel variant resides in the transactivation domain of MafB, affected by all the missense mutations (residues 54-71) described to date (Fig. 2C) (7).

Variable phenotypes and even intra-familial variability was observed in the probands investigated by Zankl et al (7). This suggests a role for as yet unidentified modifier gene(s), epigenetic mechanisms or environmental factors in disease penetrance which could also explain the absence of the associated renal phenotype. Gender differences in penetrance due to a protective modifier gene may also be an explanation for the presence of three healthy females in our family. Actually, incomplete penetrance occurring only in female carriers has been described in familial hypokalemic periodic paralysis due to mutations in the skeletal muscle calcium channel $\alpha 1$ subunit gene (16). Also, asymptomatic females carrying the same survival motor neuron 1 gene mutation as their affected relatives with recessive spinal muscular atrophy have been observed (17). On the other hand, such a protective effect in females should result in an unequal gender ratio. However, a review of the data of all MCTO patients reported to date showed no male predominance of the disease.

Similar to the data presented, several other bone diseases exhibit variable expressivity and incomplete penetrance. For example, heterozygous mutations in the sequestosome (SQSTM)1 gene are now suggested not to cause Paget's disease of bone (OMIM 601530) but only predispose a carrier to the disease (18). The fact that a single mutant allele can be insufficient to cause the disorder has also been observed in otosclerosis 1 (OMIM \%166800) (19), characterized by alternating phases of bone resorption and formation, as well as growth differentiation factor 6-attributable oculo-skeletal phenotypes (20).

Finally, it would of interest to observe the effects of the heterozygous p.Ser56Phe and other missense mutations in a mouse model. Complete MafB deficiency has been shown to cause defective respiratory rhythmogenesis and fatal central apnea at birth (21). However, all mouse models were generated by mutations not comparable with the missense mutations observed in MCTO patients. In the first model, MafB/ Kreisler (kr), MAFB has been shown to be the ortholog of the gene affected by the mouse Kreisler mutation, that involves a submicroscopic inversion, leaving $30 \mathrm{~kb}$ of $5^{\prime}$ sequence and the coding region intact $(8,22)$. Of note, in these homozygous mice, the expression of the $M a f B / k r$ gene is still maintained outside of the hindbrain indicating a selective, tissue-specific gene inactivation (22). Using N-ethyl-N-nitrosourea (ENU), Cordes and Barsh (8) introduced an A-to-G transition at nucleotide 743 resulting in a p.Asp248Ser substitution. The mutation is located in the putative DNA-binding domain and there seems to be a similar effect of the $k r^{E N U}$ mutation which is suggested to retain partial function in the absence of DNA binding, possibly through interactions with other bZip transcription (co)factors (13). A previous study demonstrated that $k r^{E N U}$ was able to form dimers with WT MafB and transactivated a reporter gene (23). Finally, a targeting vector (MafB/green fluorescent protein) was designed and RT-PCR failed to detect transcripts in mutant renal cells and macrophages (13). Again, all $\mathrm{MafB}^{-/-}$ mice died within $24 \mathrm{~h}$ after birth. A phenotype strikingly similar to that of the Kreisler mutant, was observed in these $\mathrm{MafB}^{-{ }^{-}}$neonates but not in their heterozygous siblings. Hence, it remains elusive, whether a heterozygous missense mutation in the amino-terminal transcriptional activation domain of mouse $M a f B$ mirrors the phenotype observed in humans and whether incomplete penetrance can also be observed in this model.

In conclusion, the novel $M A F B$ variant observed is expected to be responsible for the MCTO phenotype in our patient. The effect of a modifier, incomplete penetrance, epigenetic mechanisms or environmental factors may account for the variable expressivity of the disease in the family presented in our study. This not only increases the complexity of genetic counseling but should also be considered in patient care, at least in renal prognosis.

\section{Acknowledgements}

We thank the family for their kind participation in this study. We thank Pia Uerdingen for her excellent technical assistance. G.C.D. was supported by the University of Bonn, BONFOR (grant no. O-149.0096).

\section{References}

1. Jackson JBS: A boneless arm. Boston Med Surg J 18: 368-369, 1838.

2. Tyler T and Rosenbaum HD: Idiopathic multicentric osteolysis. AJR Am J Roentgenol 126: 23-31, 1976.

3. Hall M: International nosology and classification of constitutional disorders of bone (2001). Am J Med Genet 113: 65-77, 2002.

4. Faber MR, Verlaak R, Fiselier TJ, Hamel BC, Franssen MJ and Gerrits GP: Inherited multicentric osteolysis with carpal-tarsal localisation mimicking juvenile idiopathic arthritis. Eur J Pediatr 163: 612-618, 2004.

5. Pai GS and Macpherson RI: Idiopathic multicentric osteolysis: report of two new cases and review of the literature. Am J Med Genet 29: 929-936, 1988.

6. Carnevale A, Canún S, Mendoza L and del Castillo V: Idiopathic multicentric osteolysis with facial anomalies and nephropathy. Am J Med Genet 26: 877-886, 1987.

7. Zankl A, Duncan EL, Leo PJ, Clark GR, Glazov EA, Addor MC, Herlin T, Kim CA, Leheup BP, McGill J, et al: Multicentric carpotarsal osteolysis is caused by mutations clustering in the amino-terminal transcriptional activation domain of $M A F B$. Am J Hum Genet 90: 494-501, 2012. 
8. Cordes SP and Barsh GS: The mouse segmentation gene $\mathrm{kr}$ encodes a novel basic domain-leucine zipper transcription factor. Cell 79: 1025-1034, 1994.

9. Sadl V, Jin F, Yu J, Cui S, Holmyard D, Quaggin S, Barsh G and Cordes S: The mouse Kreisler (Krml1/MafB) segmentation gene is required for differentiation of glomerular visceral epithelial cells. Dev Biol 249: 16-29, 2002.

10. Nishimura W, Kondo T, Salameh T, El Khattabi I, Dodge R, Bonner-Weir S and Sharma A: A switch from MafB to MafA expression accompanies differentiation to pancreatic beta-cells. Dev Biol 293: 526-539, 2006.

11. Artner I, Blanchi B, Raum JC, Guo M, Kaneko T, Cordes S Sieweke $\mathrm{M}$ and Stein R: MafB is required for islet beta cell maturation. Proc Natl Acad Sci USA 104: 3853-3858, 2007.

12. Kim K, Kim JH, Lee J, Jin HM, Kook H, Kim KK, Lee SY and Kim N: MafB negatively regulates RANKL-mediated osteoclast differentiation. Blood 109: 3253-3259, 2007.

13. Moriguchi T, Hamada M, Morito N, Terunuma T, Hasegawa K, Zhang C, Yokomizo T, Esaki R, Kuroda E, Yoh K, et al: MafB is essential for renal development and F4/80 expression in macrophages. Mol Cell Biol 26: 5715-5727, 2006.

14. Draaken M, Giesen CA, Kesselheim AL, Jabs R, Aretz S, Kugaudo M, Chrzanowska KH, Krajewska-Walasek M and Ludwig M: Maternal de novo triple mosaicism for two single $O C R L$ nucleotide substitutions (c.1736A $>\mathrm{T}, \mathrm{c} .1736 \mathrm{~A}>\mathrm{G})$ in a Lowe syndrome family. Hum Genet 129: 513-519, 2011.

15. Hezard N, Cornillet P, Droulle C, Gillot L, Potron G and Nguyen P: Factor V Leiden: detection in whole blood by ASA PCR using an additional mismatch in antepenultimate position. Thromb Res 88: 59-66, 1997.

16. Kawamura S, Ikeda Y, Tomita K, Watanabe N and Seki K: A family of hypokalemic periodic paralysis with CACNA1S gene mutation showing incomplete penetrance in women. Intern Med 43: 218-222, 2004.
17. Oprea GE, Kröber S, McWhorter ML, Rossoll W, Müller S, Krawczak M, Bassell GJ, Beattie CE and Wirth B: Plastin 3 is a protective modifier of autosomal recessive spinal muscular atrophy. Science 320: 524-527, 2008

18. Leach RJ, Singer FR, Ench Y, Wisdom JH, Pina DS and Johnson-Pais TL: Clinical and cellular phenotypes associated with sequestosome 1 (SQSTM1) mutations. J Bone Miner Res 21 (Suppl 2): P45-P50, 2006.

19. Uppal S, Bajaj Y, Rustom I and Coatesworth AP: Otosclerosis 1: the aetiopathogenesis of otosclerosis. Int J Clin Pract 63 1526-1530, 2009.

20. Asai-Coakwell M, French CR, Ye M, Garcha K, Bigot K, Perera AG, Staehling-Hampton K, Mema SC, Chanda B, Mushegian A, et al: Incomplete penetrance and phenotypic variability characterize Gdf6-attributable oculo-skeletal phenotypes. Hum Mol Genet 18: 1110-1121, 2009.

21. Blanchi B, Kelly LM, Viemari JC, Lafon I, Burnet H, Bévengut M, Tillmanns S, Daniel L, Graf T, Hilaire G and Sieweke MH: MafB deficiency causes defective respiratory rhythmogenesis and fatal central apnea at birth. Nat Neurosci 6: 1091-1099, 2003.

22. Eichmann A, Grapin-Botton A, Kelly L, Graf T, Le Douarin NM and Sieweke M: The expression pattern of the $\mathrm{mafB} / \mathrm{kr}$ gene in birds and mice reveals that the kreisler phenotype does not represent a null mutant. Mech Dev 65: 111-122, 1997.

23. Sadl VS, Sing A, Mar L, Jin F and Cordes SP: Analysis of hindbrain patterning defects caused by the kreisler $^{\text {enu }}$ mutation reveals multiple roles of Kreisler in hindbrain segmentation. Dev Dyn 227: 134-142, 2003. 